\title{
Aplicación de Internet de las cosas en el monitoreo de la producción de lombricomposta
}

\section{Application of the Internet of things in the monitoring of the production of vermicompost}

\author{
ALANIS-TEUTLE, Raúl†*, RAMIREZ-CHOCOLATL, Yuridia, ALONSO-CALPEÑO, Mariela Juana \\ y SANTANDER-CASTILLO, Julieta
}

Instituto Tecnológico Superior de Atlixco, División de la Ingeniería en Sistemas Computacionales

ID $1^{\text {er }}$ Autor: Raúl, Alanis-Teutle / ORC ID: 0000-0003-1852-1149

ID $1^{\text {er }}$ Coautor: Yuridia, Ramirez-Chocolatl / ORC ID: 0000-0002-7840-0982

ID $2^{\text {do }}$ Coautor: Mariela Juana, Alonso-Calpeño / ORC ID: 0000-0001-7276-1923

ID $3^{\text {er }}$ Coautor: Julieta, Santander-Castillo / ORC ID: 0000-0002-6998-471X

DOI: $10.35429 /$ JCT.2019.10.3.23.32

Recibido 30 de Junio, 2019, Aceptado, 07 de Septiembre, 2019

\section{Resumen}

En el presente artículo se muestran los resultados de la aplicación de una arquitectura basada en Internet de las cosas para la producción de lombricomposta a través de una interfaz que permite monitorear y recolectar los datos de temperatura, humedad, y $\mathrm{pH}$ mediante sensores inteligentes que utilizan el servicio de cómputo en la nube. La metodología utilizada para la construcción de la arquitectura, se basó en una búsqueda del estado del arte en bases de datos abiertas. Para la implementación de la misma, se construyó un experimento puro con una variable dependiente y dos independientes. Los resultados obtenidos por las mediciones realizadas durante un periodo de cuatro meses, permitieron crear gráficas donde se observan los cambios constantes de temperatura en un rango de 19 a $27^{\circ} \mathrm{C}$, la humedad en el rango de $41 \%$ a $85 \%$ y el $\mathrm{pH}$ de 5 a 8.4 en el ácido del sustrato. Dicha información obtenida de manera ubicua, ayudó al usuario a generar un plan semanal de riego, aireación, y mezcla de la lombricomposta, con el fin de reducir tiempos, costos y esfuerzo humano. Asimismo, ayudó a mantener las condiciones ambientales óptimas para la reproducción de las lombrices.

IoT, Lombricomposta, Nube

\begin{abstract}
In this article we show the results of the application of an architecture based on Internet of things for the production of vermicompost through an interface that allows to monitor and collect temperature, humidity, and $\mathrm{pH}$ data through intelligent sensors that use the cloud computing service. The methodology used for the construction of the architecture was based on a search of the state of the art in open databases. For the implementation of the same, a pure experiment with a dependent variable and two independent ones was built. The results obtained by the measurements made during a period of four months, allowed to create grapHs where the constant temperature changes are observed in a range of 19 to $27^{\circ} \mathrm{C}$, humidity in the range of $41 \%$ to $85 \%$ and the $\mathrm{pH}$ of 5 to 8.4 in the acid of the substrate. This information obtained in a ubiquitous way, helped the user to generate a weekly plan for irrigation, aeration, and mixing of the vermicompost, in order to reduce time, costs and human effort. It also helped maintain optimal environmental conditions for the reproduction of earthworms.
\end{abstract}

Internet of things, Vermicompost, Cloud computing

Citación: ALANIS-TEUTLE, Raúl, RAMIREZ-CHOCOLATL, Yuridia, ALONSO-CALPEÑO, Mariela Juana y SANTANDER-CASTILLO, Julieta. Aplicación de Internet de las cosas en el monitoreo de la producción de lombricomposta. Revista de Tecnología Informática. 2019 3-10: 23-32

\footnotetext{
* Correspondencia del Autor (Correo electrónico: raul.alanis @itsatlixco.edu.mx)

$\dagger$ Investigador contribuyendo como primer autor.
} 


\section{Introducción}

El desarrollo creciente de una gran cantidad de aplicaciones para Internet de las Cosas (IoT, por sus siglas inglés Internet of Things) proporciona una amplia gama de oportunidades para crear valor agregado en diversos sectores de la industria y usuarios finales. En una de las publicaciones de Cisco, pronostica que había alrededor de 25 mil millones de objetos conectados en todo el mundo en 2015, y este número de objetos puede duplicarse para el año 2020. (Evans, 2011)

Así mismo Karen Rose, Scott Eldridge, Lyman Chapin comentan que las proyecciones del impacto de la IoT sobre Internet y la economía son impresionantes y hay quienes anticipan que en el año 2025 habrá hasta cien mil millones de dispositivos conectados a la IoT y que su impacto será de US\$11.000.000.000.000. (Karen Rose, 2015)

Por otra parte, la tecnología de Internet de las cosas se basa en la conexión de objetos cotidianos a Internet que intercambian, agregan y procesan información sobre su entorno físico para proporcionar servicios de valor añadido a los usuarios finales. Para unir estos componentes dinámicos de la infraestructura específica de la IoT se debe adoptar una arquitectura emergente con múltiples niveles que facilite el enfoque sistemático de las soluciones de IoT. (Gerber, 2017)

La IoT es una arquitectura emergente basada en la Internet global técnica facilitando el intercambio de mercancías en una red de cadena de suministro mundial. A medida que la tendencia de la tecnología se desplaza a velocidades de datos más rápidas y menor latencia de conectividad, se espera que Internet duplique su tamaño cada 3 años y la computación en la nube puede jugar un papel clave en ese crecimiento. (Ovidiu Vermesan, 2014)

(Mell \& Grance, 2011) define el cómputo en la nube como: Un modelo para permitir el acceso ubicuo a la red, conveniente y bajo demanda a un conjunto de recursos informáticos configurables (por ejemplo, redes, servidores, almacenamiento, aplicaciones y servicios) que se puede aprovisionar y lanzar rápidamente con un mínimo esfuerzo de gestión o interacción del proveedor de servicios.
El número de aplicaciones y servicios que pueden proporcionar es prácticamente ilimitado y se puede adaptar a muchos campos de la actividad humana, facilitando y mejorando su calidad de vida en múltiples formas. Algunas de las aplicaciones y servicios IoT son: ciudades inteligentes, transporte, educación, teléfonos inteligentes, salud, servicios de energía, fabricación, compras, agricultura y medio ambiente.

El proyecto está dirigido al sector agrícola, el cual se enfoca a la siembra de todo tipo de árboles, hortalizas, plantas de ornato, para que esto se realice es necesario utilizar fertilizantes. Sin embargo, un alto porcentaje de agricultores utilizan los fertilizantes químicos esto por costumbre más que por el beneficio económico o de calidad que pudiera darle al producto final.

Actualmente se está dando un impulso para la utilización de abonos orgánicos ya que estos tienen un bajo costo, además de que ayudan a conservar la fertilidad de los suelos no dañando al medio ambiente.

Derivado de lo anterior y de las aplicaciones que han surgido en diferentes sectores se tiene como objetivo desarrollar una aplicación de IoT para el monitoreo de lombricomposta.

Por lo que en este proyecto se busca aplicar las tecnologías del Internet de las cosas (IoT) a través de una interfaz que permita monitorear mediante sensores las características de temperatura, humedad, ph y aireación del proceso de la lombricomposta desde cualquier lugar donde se encuentre el usuario para la toma de decisiones durante dicho proceso. Este proyecto es desarrollado por la Ingeniería en Sistemas Computacionales donde la producción de lombricomposta se realiza de forma artesanal y durante el análisis de su producción se detectaron los siguientes problemas: plagas como la hormiga roja y roedores, cambios en la temperatura, humedad y $\mathrm{pH}$ que afectan el crecimiento de la lombriz californiana.

Los cuidados más comunes que se deben observar para mantener sano y eficiente el procesamiento con las lombrices tienen que ver con proporcionarles la temperatura, humedad, acidez, aireación, así como el alimento, en el tipo y en las cantidades adecuadas.

ALANIS-TEUTLE, Raúl, RAMIREZ-CHOCOLATL, Yuridia, ALONSO-CALPEÑO, Mariela Juana y SANTANDERCASTILLO, Julieta. Aplicación de Internet de las cosas en el monitoreo de la producción de lombricomposta. Revista de Tecnología Informática. 2019 
El presente trabajo está dividido en dos secciones, la primera sección describe los referentes teóricos acerca de la lombricomposta, Internet de las cosas, arquitectura de la IoT, y computo en la nube. En la segunda sección se aborda el resultado de la implementación de la arquitectura de IoT en cuatro capas principales aplicada al proyecto.

\section{Marco teórico}

En este apartado se describe brevemente los temas que sustentan el proyecto.

\section{Lombricomposta}

La lombricomposta es considerada un excelente mejorador de suelos debido a que da cuerpo a los suelos arenosos y/o erosionados, mejorando su drenaje y su calidad nutritiva, revitaliza y estabiliza los suelos empobrecidos por el excesivo uso de fertilizantes químicos. (Agrovida, 2015)

La lombriz que se utiliza para este proceso es la lombriz roja californiana (Eisenia fétida) es la especie de mayor popularidad en la técnica de lombricomposteo, debido a su habilidad para digerir residuos orgánicos en condiciones de cautiverio y producir humus comercializable.

La lombriz roja californiana puede consumir entre $50 \%$ y $100 \%$ de su peso diario y duplicar su población en 90 días.

Los factores principales en la formación de la lombricomposta son: lombrices.

- Temperatura óptima. $25{ }^{\circ} \mathrm{C}$, para las

- Potencial de Hidrógeno (pH). En el rango de 6.5 a 7.5 .

- Humedad. Lo más recomendable es mantenerla entre 40-50\%; lo que se considera necesario para permitir la movilidad de la lombriz entre los desechos, propiciar su fragmentación y posibilitar su respiración. (Amaro, 2015)

Para la producción de composta se pueden utilizar distintos desechos orgánicos (Valero-Soria, 2015), como son:

\section{- $\quad$ Estiércol bovino \\ - $\quad$ Desechos de jardinería \\ - Melaza \\ - $\quad$ Residuos de caña de azúcar}

Estos desechos son mezclados con tierra y manteniendo la humedad con agua.

\section{Internet de las Cosas}

El Internet de las cosas es la comunicación entre dispositivos vía Internet para el manejo de procesos utilizando sistemas embebidos, aplicaciones móviles y la nube. Haciendo uso de esto el usuario puede utilizar un dispositivo móvil para interactuar y obtener información del sistema embebido a través de la nube para el monitoreo y control de las variables. Todo esto se incluye en el nuevo concepto conocido como industria 4.0. (González-Rodríguez, 2018)

Los objetos usados en el contexto de IoT pueden ser físicos o virtuales y tienen la posibilidad de ser conectados e identificados en redes de comunicaciones y estos pueden contener información conexa que puede ser dinámica o estática. Los objetos físicos se pueden detectar, actuar sobre ellos y conectarlos un ejemplo de ellos son los robots industriales y equipos electrónicos. Los objetos virtuales son los que existen en el mundo de la información y pueden ser almacenados, procesados y se puede acceder a ellos mismos, un ejemplo son el software de aplicaciones y los contenidos multimedia. (Arévalo, 2018)

Por lo tanto, el IoT puede ser visto como una combinación de sensores y actuadores que son capaces de proporcionar y recibir información digitalizada y colocarla en redes bidireccionales capaces de transmitir todos los datos para ser utilizados por una gran cantidad de diferentes servicios y usuarios finales. (Charith Perera, 2013)

Hasta ahora el IoT consiste en 7 billones de aparatos y se espera que para el 2020 ésta cifra aumente a 50 billones. Casi la mitad de estos consumidores tendrán un aparato conectado al Internet, con lo cual el IoT será probablemente la mejor oportunidad para las industrias en un futuro, como proveedores clave para la conectividad entre los sensores, los aparatos, los centros de información y las personas.

ALANIS-TEUTLE, Raúl, RAMIREZ-CHOCOLATL, Yuridia, ALONSO-CALPEÑO, Mariela Juana y SANTANDERCASTILLO, Julieta. Aplicación de Internet de las cosas en el monitoreo de la producción de lombricomposta. Revista de Tecnología Informática. 2019 
La industria 4.0 nos plantea la posibilidad de hacer una automatización y control utilizando los diferentes componentes que conforman un proceso interconectándolos entre sí a través de Internet. (Robles-Sosa, 2018)

El Internet de las cosas proporciona soluciones basadas en la integración de tecnologías de la información, que se refiere al hardware y software utilizado para almacenar, recuperar, y procesar datos y tecnología de comunicaciones que incluye sistemas electrónicos utilizados para la comunicación entre individuos o grupos. $\mathrm{La}$ rápida convergencia de la tecnología de la información y las comunicaciones es en tres capas de innovación tecnológica: la nube, los datos y redes de comunicación más dispositivos. (Ovidiu Vermesan, 2014)

Los proyectos con IoT se caracterizan por el uso de múltiples sensores, utilización de dispositivos embebidos, nano-dispositivos, microcontrola-dores, software y hardware generalmente libre como Arduino o Raspberry. En este concepto, casi cualquier objeto puede conectarse a una red y es así como IoT tiene el reto de la heterogeneidad de dispositivos y la heterogeneidad del tráfico. El internet de las cosas debería ser capaz de interconectar trillones de objetos heterogéneos a través de internet es por tal razón la importancia de la estandarización. (Montoya Castro, Amezquita, \& Henao, 2019)

Las arquitecturas y modelos de referencia de IoT ofrecen una vista panorámica de todo el sistema subyacente, por lo que se basan en proporcionar un mejor y mayor nivel de abstracción, con características específicas y detalles de implementación. (Rajkumar Buyya, 2016)

La arquitectura tiene que cumplir ciertos requerimientos para que esta tecnología sea viable. Debe permitir que la tecnología sea distribuida, donde los objetos puedan interactuar entre ellos, escalable, flexible, robusta, eficiente y segura. A continuación, se muestran los componentes unificados para la arquitectura de la IoT:

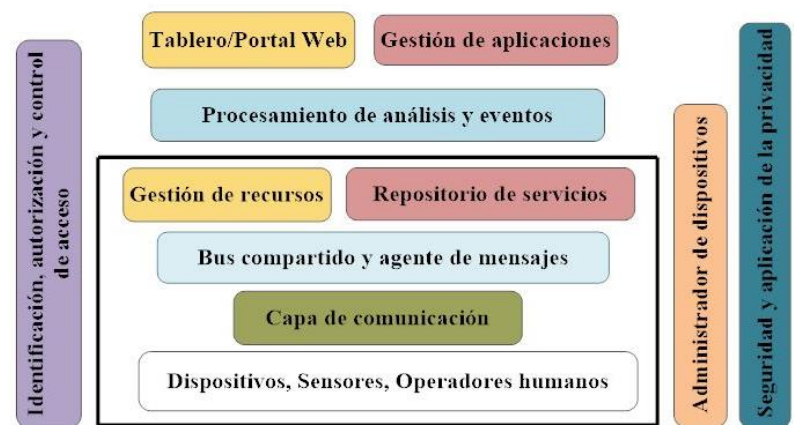

Figura 1 Componentes unificados para las arquitecturas de IoT

Fuente: Rajkumar Buyya, 2016

De manera general las arquitecturas relevantes de la IoT se dividen en diferentes módulos y estos están compuestos por capas que deben satisfacer requerimientos según su área de aplicación. Una arquitectura relevante es una herramienta esperada por un arquitecto de sistemas. Esta debería, en una forma modular mediante bloques y opciones flexibles de diseño, ser capaz de abarcar y describir sistemas específicos que no pierdan de vista los requerimientos funcionales, el desempeño, el despliegue, la estandarización de interfaces, la seguridad y la conectividad con un entorno que tiende a un esquema de movilidad IP.

En la siguiente tabla se mencionan las cuatro arquitecturas relevantes de la IoT aplicadas en la industria 4.0.

\begin{tabular}{|l|ll|}
\hline \multicolumn{1}{|c|}{ Modulo } \\
$\begin{array}{l}\text { Tres } \\
\text { capas }\end{array}$ & 1. & Capa de percepción (objetos físicos y \\
& 2ensores) \\
& 2. & Capa de red (transporte) \\
3. & Capa de aplicación \\
\hline $\begin{array}{l}\text { Cuatro } \\
\text { capas }\end{array}$ & 1. & Capa de detección \\
& 2. & Capa de intercambio de Datos \\
& 3. & Capa de integración de la información \\
4. & Capa de servicio de Aplicación \\
\hline capas & 1. & Capa de percepción \\
& 2. & Capa de transporte \\
& 3. & Capa de procesamiento \\
& 4. & Capa de aplicación \\
5. & Capa de negocios \\
\hline Siete & 1. & Capa de dispositivos, sensores \\
capas & controladores \\
& 2. & Capa de comunicación y conectividad \\
& 3. & Capa de infraestructura de la nube \\
& 4. & Capa de almacenamiento de datos \\
& 5. & Capa de análisis de datos \\
& 6. & Capa de aplicación \\
& 7. & Capa de toma de decisiones \\
\hline
\end{tabular}

Tabla 1 Módulos de las Arquitecturas de IoT Fuente: Elaboración Propia, según Rajkumar Buyya, 2016; Ovidiu Vermesan, 2014; Ray, 2018 
Las implementaciones de la IoT utilizan diferentes modelos de conectividad, cada uno de los cuales tiene sus propias características. (Karen Rose, 2015)

A continuación, se describen brevemente los cuatro modelos de conectividad:

\section{Device-to-Device (dispositivo a dispositivo): El modelo representa dos o más dispositivos que se conectan y se comunican directamente entre sí y no a través de un servidor de aplicaciones intermediario (ver figura 2 )}

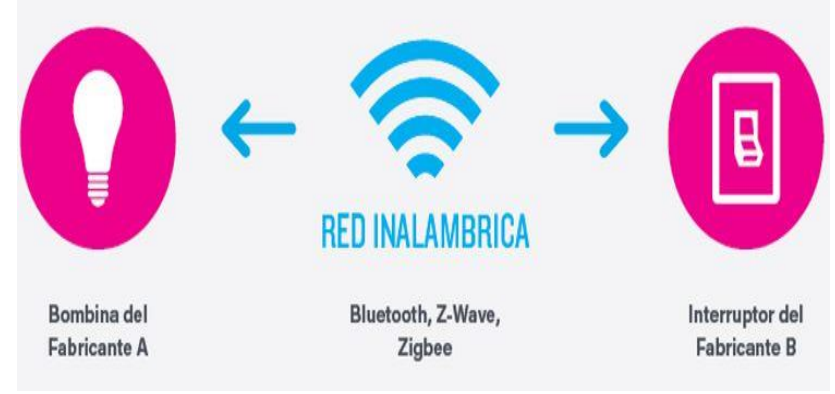

Figura 2 Comunicación dispositivo a dispositivo Fuente: Karen Rose, 2015

2. Device-to-Cloud (dispositivo a la nube): El dispositivo de la IoT se conecta directamente a un servicio en la nube, como por ejemplo un proveedor de servicios de aplicaciones para intercambiar datos y controlar el tráfico de mensajes (ver figura 3 ).

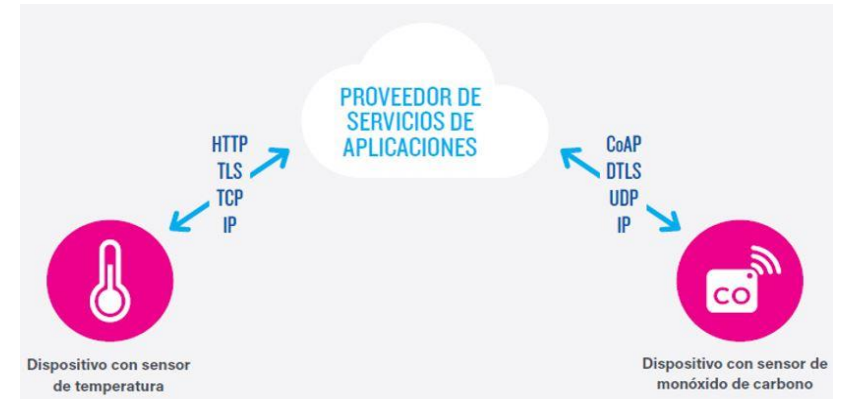

Figura 3 Ejemplo de la comunicación dispositivo a la nube

Fuente: Karen Rose, 2015

3. Device-to-Gateway (dispositivo a puerta de enlace): El modelo dispositivo a puerta de enlace de capa de aplicación (ALG), el dispositivo de la IoT se conecta a través de un servicio ALG como una forma de llegar a un servicio en la nube.

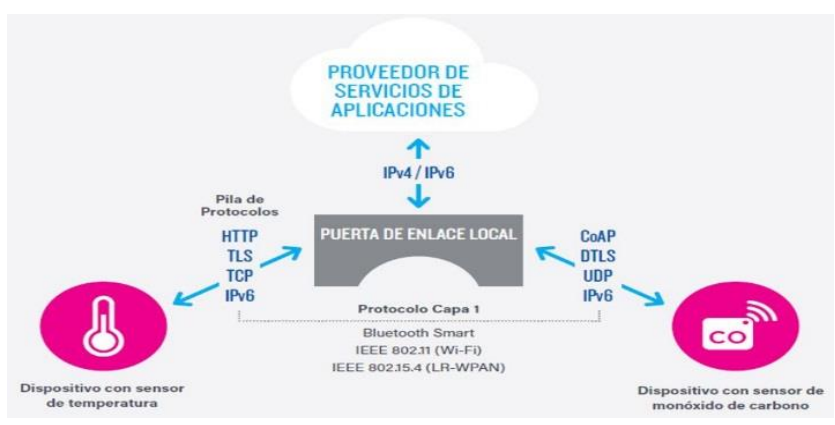

Figura 4 Comunicación dispositivo a puerta de enlace Fuente: Karen Rose, 2015

\section{Back-End Data-Sharing (intercambio de datos a través del back-end): Se refiere a una arquitectura de comunicación que permite que los usuarios exporten y analicen datos de objetos inteligentes de un servicio en la nube en combinación con datos de otras fuentes. Esta arquitectura soporta "el deseo del usuario de permitir que terceros accedan a los datos subidos por sus sensores".}

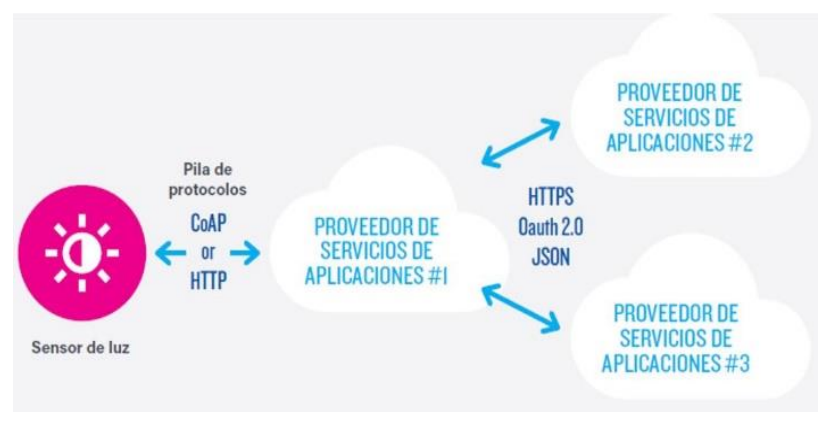

Figura 5 Intercambio de datos a través de black-end Fuente: Karen Rose, 2015

\section{Cómputo en la nube}

El cómputo en la nube es un modelo para crear acceso conveniente, ubicuo y bajo demanda, vía Internet, a un conjunto compartido de recursos de cómputo configurables (por ejemplo, redes, servidores, almacenamiento, aplicaciones y servicios), los cuales pueden ser rápidamente asignados y provistos con un mínimo de gestión administrativa e interacción con el proveedor.

Este modelo promueve la disponibilidad; tiene cinco características esenciales, tres modelos de servicio y cuatro modelos de despliegue. (Barnard, Delgado, \& Juan, 2016)

Las características esenciales, los modelos de servicio y los modelos de despliegue se muestran en la figura 6.

ALANIS-TEUTLE, Raúl, RAMIREZ-CHOCOLATL, Yuridia ALONSO-CALPEÑO, Mariela Juana y SANTANDERCASTILLO, Julieta. Aplicación de Internet de las cosas en el monitoreo de la producción de lombricomposta. Revista de Tecnología Informática. 2019 


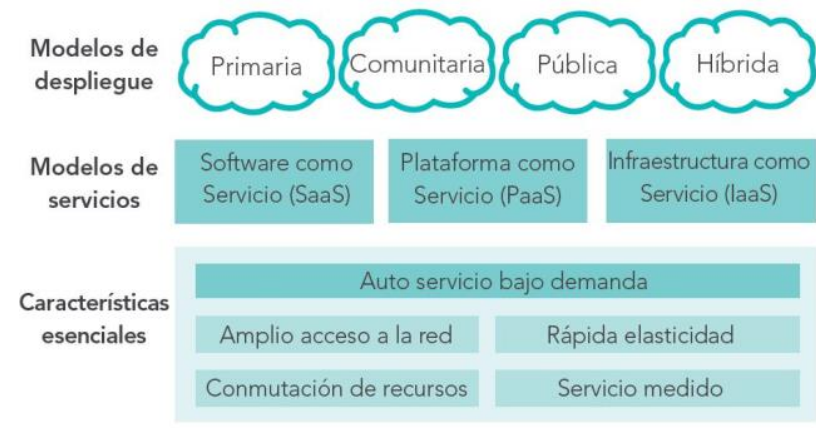

Figura 6 Modelos de cómputo en la nube Fuente: Barnard, Delgado, \& Juan, 2016

\section{Desarrollo}

El método de investigación a aplicar en el proyecto es cuantitativo, ya que permite la recolección de datos estadísticos, para este proyecto se requiere recabar información acerca de índices de temperatura, humedad y $\mathrm{pH}$ mediante sensores, y con base en ellos emitir alertas al usuario para tomar decisiones. Quedando por tanto las siguientes fases:

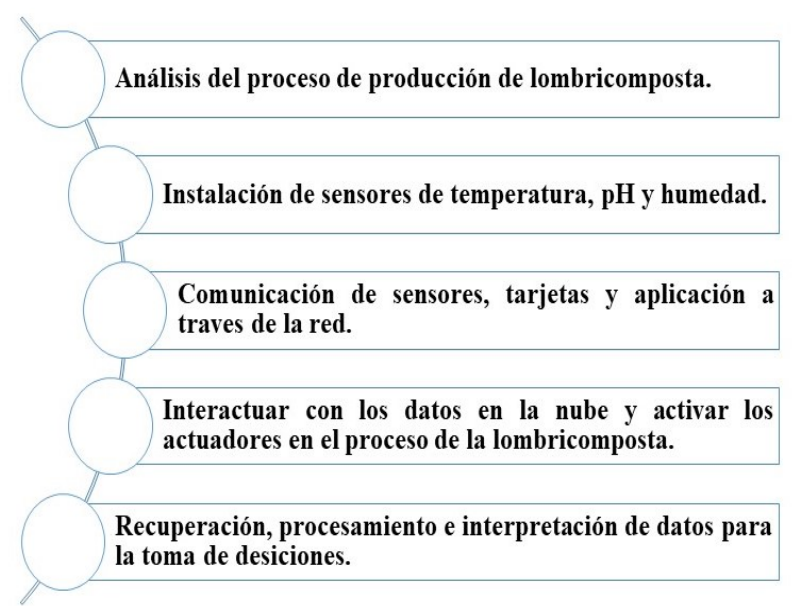

Figura 7 Fases de investigación

Fuente: Elaboración Propia

De acuerdo con las etapas de la metodología aplicada en el proyecto se determinó utilizar el módulo de la arquitectura de IoT en cuatro capas para describir las funciones de la aplicación y el papel de cada capa, en la figura 8 se muestra las capas aplicadas al proceso de producción de la lombricomposta.

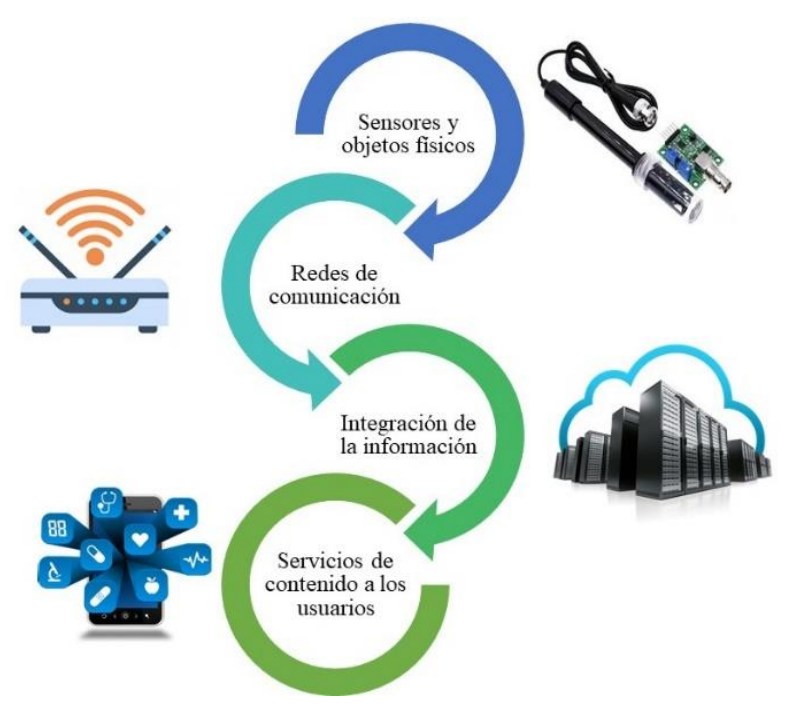

Figura 8 Arquitectura de IoT en 4 capas Fuente: Elaboración Propia

\section{Resultados}

Los resultados de la aplicación de la arquitectura de referencia basada en Internet de las cosas para la producción de lombricomposta a través de una interfaz permiten monitorear y recolectar los datos de temperatura con un rango de $25-29^{\circ} \mathrm{C}$, humedad con un rango de $40-50 \%$, y pH con un rango de 6.5 a $7.5 \%$. Mediante el uso de los sensores inteligentes que utilizan el servicio de cómputo en la nube se pueden emitir alertas en tiempo real cuando uno de los valores esta fuera de los rangos ideales de las variables monitoreadas en el proceso de lombricomposta, a continuación, se describen las actividades realizadas por cada capa.

\section{Capa de detección}

En esta capa se consideran los dispositivos IoT necesarios para tener una comunicación directa o indirecta que harán posible la conexión a Internet, los componentes de conexión directa utilizados son: Arduino mega 2560, Ethernet Shield con conexión a Internet.

Los sensores que se utilizan para obtener los datos son el sensor digital de temperatura y humedad DTH11, así como el sensor electrodo para medir $\mathrm{pH}$ conectados a la tarjeta de Arduino con conexión a la base de datos en MySQL utilizando el servicio de la herramienta de XAMPP. Con dichos datos se realiza un preprocesamiento para determinar qué datos se envían a la nube y decidir el tiempo de obtención de datos o valores fuera rango de acuerdo con los parámetros establecidos en la producción de lombricomposta. 
Para realizar la función del actuador se programaron y conectaron ventiladores al detectar un cambio en la temperatura. En la figura se muestra el resultado de los dispositivos conectados e instalados en el prototipo de lombricomposta.

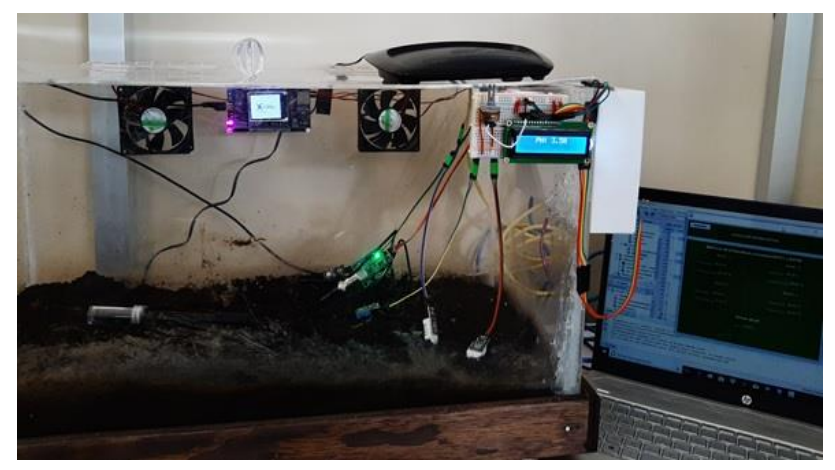

Figura 9 Prototipo de lombricomposta con la implementación IoT

Fuente: Elaboración Propia

\section{Capa de intercambio de datos}

Esta capa va dirigida a soportar la conexión de los dispositivos a la red, el cual está regido por múltiples protocolos para su comunicación ya sea entre dispositivos o a la red misma.

Esta capa dispone de las herramientas necesarias para transmitir datos entre dispositivos (o servidores y dispositivos de red), y también para realizar cierto grado de procesamiento de los mismos.

A través del protocolo TCP/IP se controla la transmisión de los datos mediante la placa Ethernet Shield conectada a la placa Arduino asignando una dirección IP mediante el protocolo DHCP del Router, este deberá ser configurado previamente para determinar el rango de IP's a utilizar, la función de la tarjeta Shield es conectar el dispositivo al Router encargado de la transición de la información a la nube mediante la puerta de enlace.

\section{Capa de integración de la información}

El modelo de conectividad aplicado al proyecto es dispositivo a la nube, debido a que los dispositivos de la IoT se conectan directamente al servicio de la base de datos en tiempo real de Firebase para guardar los datos necesarios de la temperatura, humedad y $\mathrm{pH}$ que se requieren mostrar y graficar en la aplicación.
Al procesar esos datos se pueden consultar a través de la aplicación conectada a la base de datos, así que se puede decir que esta es una de las capas principales de la arquitectura de IoT del proyecto.

El procesamiento de la información y filtrado de los datos no deseados e integración de la información principal es útil para los usuarios finales. Debido a que se tienen que tomar medidas en el proceso de riego, aireación y mezcla de la lombricomposta, dichas medidas permiten evitar la muerte de las lombrices californianas, así como la disminución de su reproducción y población.

\section{Capa de servicio de aplicación}

Los datos recabados por los dispositivos físicos se transmiten hacia otros dispositivos 0 elementos de la red y se procesan en la aplicación final para un uso concreto de los datos.

La aplicación para el monitoreo de lombricomposta proporciona seguridad al productor ya que ninguna persona ajena podrá acceder a ella sin la autorización de este, una vez ingresados los datos del productor, usuario y contraseña este podrá ingresar a la aplicación como se muestra en la figura 10.

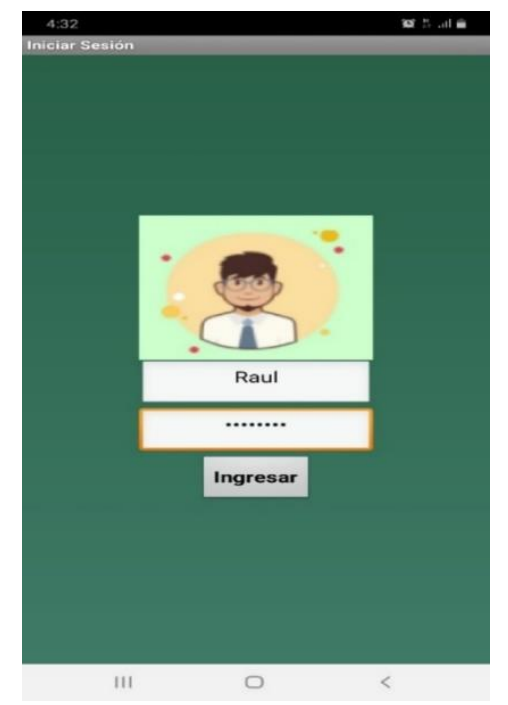

Figura 10 Inicio de sesión de la aplicación Fuente: Elaboración Propia

Una vez validada la información del productor (usuario y contraseña) la aplicación muestra los valores en tiempo real de humedad, temperatura y $\mathrm{pH}$, dichos valores son el último registro obtenidos por los sensores conectados en el prototipo de la lombricomposta.

ALANIS-TEUTLE, Raúl, RAMIREZ-CHOCOLATL, Yuridia, ALONSO-CALPEÑO, Mariela Juana y SANTANDERCASTILLO, Julieta. Aplicación de Internet de las cosas en el monitoreo de la producción de lombricomposta. Revista de Tecnología Informática. 2019 
En la figura 11 se observa que los valores están fuera de rango, lo que ocasiona que se emita una alerta y se active los ventiladores conectados al prototipo.

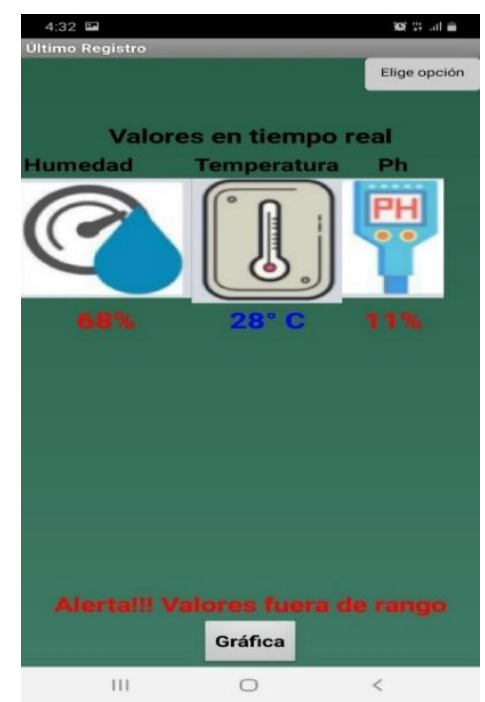

Figura 11 Valores en tiempo real Fuente: Elaboración Propia

En la figura 12 se muestran las opciones que tiene el usuario permitido realizar, como son consultas por rango de valores y conocer información de la lombricomposta.

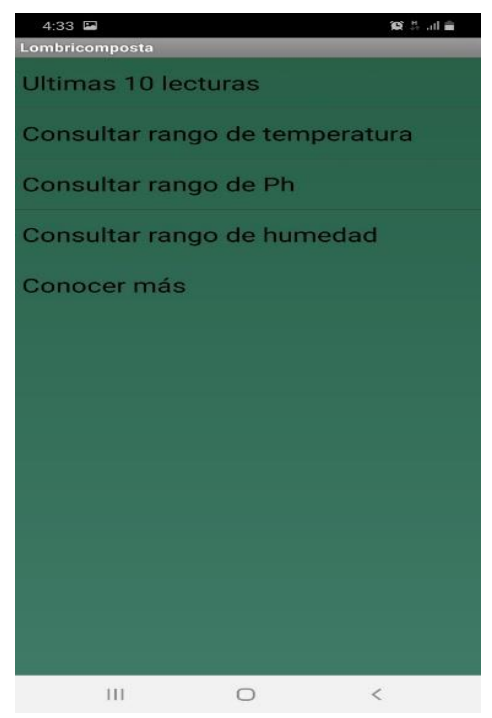

Figura 12 Consulta de información Fuente: Elaboración Propia

Al seleccionar la opción de conocer más, se muestra información general de la lombricomposta con los factores principales que debe conocer el usuario para la formación de esta. (Ver figura 13)

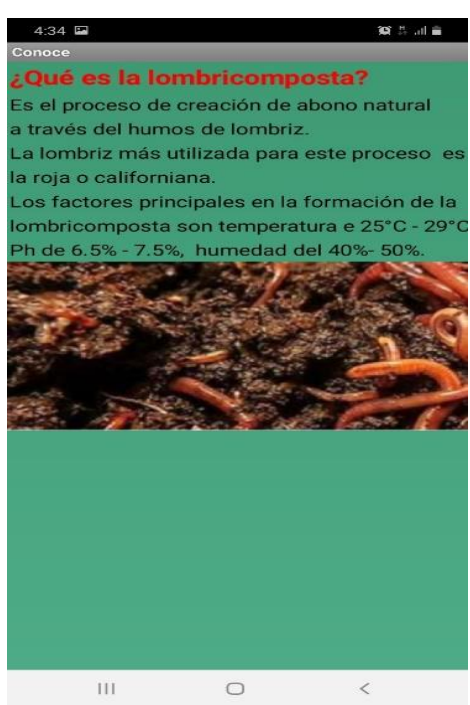

Figura 13 Información de lombricomposta Fuente: Elaboración Propia

Al realizar la consulta de valores, el usuario debe seleccionar la variable que quiere conocer e ingresar el rango a consultar. En la figura 14 se observan los datos de los registros que cumplen con la condición del $\mathrm{pH}$ en un rango de 6 y $10 \%$ de acidez. Así mismo se puede observar los valores de las variables de temperatura y humedad obtenidos de la lombricomposta en un tiempo determinado por el usuario.

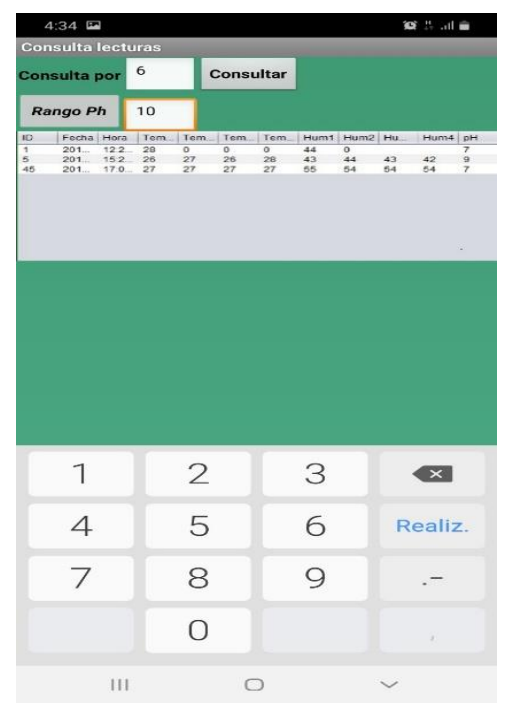

Figura 14 Consultar lecturas de datos Fuente: Elaboración Propia

Otra opción que tiene el usuario en la aplicación es poder generar y descargar la gráfica de las variables de temperatura y humedad extraídas de la base de datos en la nube, en un tiempo predefinido, como se muestra en la figura 15. Sumado a lo anterior se puede observar la variación de los valores de las variables, estando estas en el rango ideal para la producción de la lombricomposta.

ALANIS-TEUTLE, Raúl, RAMIREZ-CHOCOLATL, Yuridia, ALONSO-CALPEÑO, Mariela Juana y SANTANDERCASTILLO, Julieta. Aplicación de Internet de las cosas en el monitoreo de la producción de lombricomposta. Revista de Tecnología Informática. 2019 


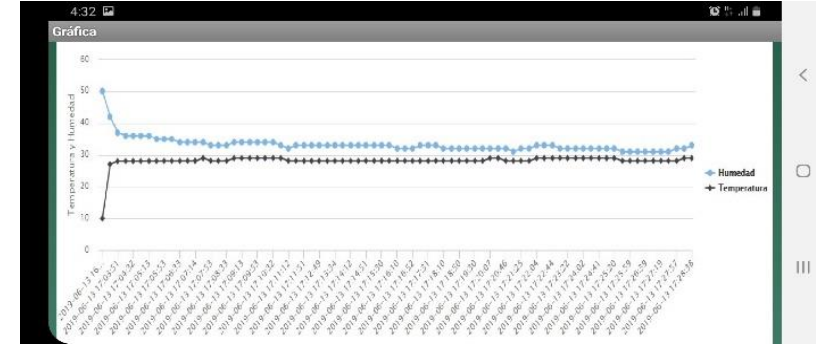

Figura 15 Gráfica de resultados Fuente: Elaboración Propia

Los resultados obtenidos por las mediciones realizadas durante un periodo de cuatro meses, permitieron crear gráficas donde se observan los cambios constantes de temperatura en un rango de 19 a $27{ }^{\circ} \mathrm{C}$, la humedad en el rango de $41 \%$ a $85 \%$ y el pH de 5 a 8.4 en el ácido del sustrato.

Dichas gráficas sirven de ayuda para tomar la decisión de realizar dos veces por semana los riegos y mezcla de la lombricomposta, así como la aireación, evitando tener malos olores y plagas. Durante este proceso se observa que la lombriz californiana se reproduce más de una vez por semana y de cada acoplamiento resultan 2 cocones de cada una de la lombriz, conteniendo cada cocón de 2 a 4 lombrices. Así mismo se observó que la lombriz va cambiando de color rosado a café obscuro y la mayoría mide entre $7 \mathrm{~cm}$. a $11 \mathrm{~cm}$., e incrementando su población cada mes.

\section{Agradecimientos}

Al Tecnológico Nacional de México, al Instituto Tecnológico Superior de Atlixco, en especial a los estudiantes y a la academia de la Ingeniería en Sistemas Computacionales, por el apoyo brindado en el desarrollo del proyecto.

\section{Conclusiones}

Como se menciona en el artículo el Internet de las cosas ha tenido un impacto en los diversos sectores de la industria, por lo que se puede concluir que la implementación de dispositivos como sensores y actuadores permiten almacenar, recuperar y procesar datos con tecnologías de comunicaciones entre individuos o grupos, los cuales proporcionan una gran ventaja al trabajar con ellos, ya que no es necesaria la interacción humana para realizar una acción, aunado a que se pueden monitorear datos en forma remota permitiendo la toma de decisiones y proporcionando servicios de valor añadido a los usuarios finales.
El uso de la tecnología y la arquitectura de 4 capas de Internet de las cosas, así como el modelo de conectividad de dispositivo a la nube aplicados en la producción de lombricomposta es ideal para el monitoreo de procesos en tiempo real lo que permite tomar acciones determinadas por el usuario final, con el fin de evitar pérdidas económicas, esfuerzo humano y reducción de la población de la lombriz. Con estas acciones se puede generar más cantidad de composta $\mathrm{y}$ obtener un fertilizante natural ideal para cualquier tipo de planta.

El prototipo de la lombricomposta y la aplicación móvil que se describen cumplen con el objetivo planteado, recuperar los datos de humedad, temperatura y $\mathrm{pH}$, con estos datos obtenidos se puede realizar una acción que permita la producción óptima de lombricomposta. Además de integrar diferentes áreas de conocimiento como son Internet de las cosas, cómputo en la nube, sistemas embebidos y aplicaciones móviles.

Sin embargo, al proyecto se le pueden adaptar e implementar la capa de negocio y la capa de toma de decisiones de la arquitectura emergente de siete capas de internet de las cosas, que permitan gestionar el proceso de la lombricomposta y aportar información al sector agrícola.

\section{Referencias}

Agrovida, A. (2015). AGROVIDA. Obtenido de http://agrovida.com.mx/lombricomposta.html

Amaro, L. M. (2015). Tratamiento de residuos sólidos orgánicos. Ciencia y Desarrollo.

Arévalo, J. A. (2018). Prototipo de un Sistema de Monitoreo de Calidad del agua subterránea en instalaciones de captación de una localidad Rural del Municipio de Tibaná - Boyacá. Bogotá, Colombia.

Barnard, A., Delgado, A., \& Juan, V. (2016). Introducción al cómputo en la nube. México: ICA/InterPARES.

Charith Perera, A. Z. (2013). Sensing as a Service Model for Smart Cities Supported by Internet of Things. Computers and Society, 8193.

ALANIS-TEUTLE, Raúl, RAMIREZ-CHOCOLATL, Yuridia, ALONSO-CALPEÑO, Mariela Juana y SANTANDERCASTILLO, Julieta. Aplicación de Internet de las cosas en el monitoreo de la producción de lombricomposta. Revista de Tecnología Informática. 2019 
Evans, D. (Abril de 2011). "The internet of things: How the next evolution of the internet is changing Everything." . Obtenido de http://www.cisco.com/c/dam/en_us/about/ac79/ docs/innov/IoT_IBSG_0411FINAL.pdf

Gerber, A. (04 de Octubre de 2017). Simplifique el desarrollo de sus soluciones de IoT con arquitecturas de IoT . Obtenido de IBM: https://www.ibm.com/developerworks/ssa/libra ry/iot-lp201-iot-architectures/index.html

González-Rodríguez, A. G.-B.-H. (2018). Automatización de una caldera pirotubular. Revista de Ingeniería Mecánica, 12-17.

Karen Rose, S. E. (Octubre de 2015). La Internet de las cosas-Una breve reseña. Obtenido de https://www.internetsociety.org/wpcontent/uploads/2017/09/reportInternetOfThings-20160817-es-1.pdf

Mell, P., \& Grance, T. (Septiembre de 2011). The NIST Definition of Cloud. Obtenido de National Institute of Standards and Technology: https://nvlpubs.nist.gov/nistpubs/Legacy/SP/nis tspecialpublication800-145.pdf

Montoya Castro, Á. M., Amezquita, J. C., \& Henao, C. R. (2019). Caracterización de la Línea de Investigación Redes Informáticas y Software del Centro de Gestión de Mercados Logística y Tecnologías de la Información. Revista especializada en tecnologíasTransversales de la organización, 46-60.

Ovidiu Vermesan, P. F. (2014). Internet of Things - From Research and Innovation to Market Deployment. River Publishers.

Rajkumar Buyya, A. V. (2016). Internet of Things Principles and Paradigms. Rajkumar Buyya, Amir Vahid Dastjerdi Elsevier .

Ray, P. (2018). A survey on Internet of Things architectures. Computer and Information Sciences, 291-319.

Robles-Sosa, A. J.-B.-H.-C. (2018). Automatización de una mezcladora didáctica utilizando el internet de las cosas para su monitoreo. Revista de Aplicaciones de la Ingeniería, 1-8.
Valero-Soria, H. A.-R.-H.-C. (2015). Producción de compostas con desechos orgánicos para el aprovechamiento de agua en el suelo. Revista de Ciencias Naturales y Agropecuarias, 195-199. 